\title{
Fabricación y caracterización de vigas dúo reforzadas de maderas de "Populus sp." y "Pinus pinaster Ait."
}

Manufacture and characterization of reinforced duo timber beams of "Populus sp." and "Pinus pinaster Ait.

Balmori Roiz, J.A. ${ }^{*}$

\begin{abstract}
'Departamento de Construcciones Arquitectónicas, Ingeniería del Terreno, Mecánica de los Medios Continuos y Teoría de Estructuras. Escuela Técnica Superior de Arquitectura. Universidad de Valladolid Avda. Salamanca 18, 47014 Valladolid (España)
\end{abstract}




\title{
Resumen
}

Esta tesis doctoral pretende desarrollar un producto industrializado de madera reforzada que permita emplear con usos estructurales maderas de rápido crecimiento y bajas prestaciones mecánicas, o de baja calidad, actualmente rechazadas. La actual exigencia social de realizar construcciones sostenibles y eficientes energéticamente, recogida en la directiva europea 2018/844/EU, posiciona a la madera como uno de los materiales de construcción con mayor proyección de crecimiento en el futuro cercano. Sin embargo, las especies gestionadas con fines de uso estructural son limitadas y sus masas forestales, pese a someterse a una gestión ordenada y planificada, son finitas en extensión y su explotación se ve condicionada por los tiempos de crecimiento propios de cada especie. Por ello, para dar respuesta a la creciente demanda de madera de un modo sostenible en el tiempo, es preciso introducir en la industria maderera nuevas especies de rápido crecimiento y, además, optimizar los recursos de las especies tradicionales. Las bajas prestaciones mecánicas de las maderas de rápido crecimiento, y la influencia negativa de las singularidades propias de la madera en aquellas de calidad rechazo, limitan actualmente su aplicación intensiva en usos estructurales. La fabricación de productos de madera industrializada reforzada con materiales polímeros reforzados con fibras (FRP) introducidos en su línea de encolado permite aumentar las prestaciones mecánicas de estas maderas, aumentando la rigidez, para posibilitar su aplicación en la construcción y rehabilitación de estructuras de edificación. En esta investigación se han utilizado dos especies de madera: chopo (Populus x euroamericana I-214) y pino pinaster (Pinus pinaster Ait.), presentes de forma extensa en toda Castilla y León y gestionadas artificialmente por el hombre en plantaciones intensivas en zonas de ribera (chopo) o en reforestaciones de terrenos áridos (pino pinaster). El cambio de uso de estas especies y la fabricación en industrias locales de un producto de segunda transformación puede generar unos nuevos recursos económicos, facilitando la creación de empleo "verde" y la fijación de población en zonas rurales.

Palabras clave: Populus x euroamericana I-214; Pinus pinaster Ait.; Refuerzos FRP; Vigas dúo.

\begin{abstract}
This doctoral thesis aims to develop a new industrialized reinforced timber product using local, fast-growing and low performance wood, or low quality, which is currently rejected for structural use. Current social requirements for sustainable and energy efficient buildings in European Directive 2018/844/EU position timber as one of the construction materials with the greatest growing potential in the future. In Europe today, traditional structural timber species are grown in well managed forests, but their harvesting capacity is conditioned by the growth times of each species and their limited surface. Therefore, to respond to the growing demand for timber in a sustainable way over time, it is necessary to introduce new fast-growing wood species into the timber industry and, in addition, to optimize the resources of traditional species. The low mechanical performance of fast-growing woods, and the negative influence of its peculiarities in the rejection timber, currently limit its intensive application to structural uses. The manufacture of industrialized timber products with Fiber Reinforced Polymer (FRP). introduced in the glue-line aims to increase their mechanical performance, focusing on improving stiffness, to allow for their introduction in the construction of building structures. In this research, two wood species have been assessed: poplar (Populus x euroamericana I-214) and maritime pine (Pinus pinaster Ait.), grown extensively throughout Castilla y León and cultivated in intensive plantations in riparian zones (poplar) or in reforestations of arid lands (maritime pine). From a social point of view, a change of use of these species and the manufacture involved may generate new economic resources, facilitating the creation of "green" employment and the establishment of population in rural areas.
\end{abstract}

Keywords: Populus x euroamericana I-214; Pinus pinaster Ait.; FRP reinforcements; Duo timber beams. 


\section{Introducción}

La madera ha sido tradicionalmente utilizada en construcción por sus excelentes propiedades mecánicas, gran disponibilidad en nuestra latitud, ligereza y facilidad de trabajo. Además, es el único material de origen completamente biológico y natural utilizado aún en la construcción, siendo un material totalmente renovable y sostenible ambientalmente (Lawson, 1996). Todo ello explica su empleo permanente en la historia de la construcción y el atractivo que sigue presentando para los estándares de construcción sostenible y energéticamente eficiente que la sociedad está exigiendo hoy en día.

La tendencia actual de la construcción con madera se encamina hacia una sustitución de la madera aserrada tradicional por diferentes productos industrializados — madera industrializada - de aplicación estructural. Productos transformados como la madera laminada encolada (MLE), el tablero contralaminado (CLT), o incluso madera reconstituida (PSL o LSP), compiten en precio y prestaciones con el resto de los materiales estructurales. Algunos de estos nuevos materiales permiten, al menos teóricamente, la fabricación de secciones con formas y dimensiones ilimitadas, y con controles de calidad intensivos que reducen la variabilidad de sus propiedades, consustancial al empleo de un material de origen natural (Thelandersson et al., 2003). Además, la introducción en la industria de máquinas de corte por control numérico (CNC), compatibles con los actuales programas de diseño gráfico y cálculo, han contribuido al desarrollo de estructuras capaces de transmitir mayores cargas con una precisión y fiabilidad superior (Willmann et al., 2016). De este modo, la madera se ha convertido en un material altamente atractivo para arquitectos e ingenieros, aumentando exponencialmente su demanda, y obligando a las grandes empresas madereras a gestionar toda la cadena de producción, empleando técnicas de silvicultura adecuadas -siembra, gestión forestal, tratamientos culturales, aserrado, y laminación-, con el fin de garantizar una producción de calidad (Brazier, 1977) y cantidad suficiente para hacer frente a la demanda de madera actual y futura. Esto ha provocado también que desde el propio sector se esté colaborando intensamente con centros de investigación para tratar de introducir en la fabricación de productos transformados maderas de rápido crecimiento y bajas prestaciones, actualmente sin uso, (Castro et al., 2003) — principalmente Salicaceaes y Myrtaceaes - , y de optimizar el uso de las maderas de menor calidad, rechazadas bajo los estándares actuales (Johansson et al., 1994).

\subsection{Contexto socioeconómico}

El cambio del modelo productivo y la industrialización del sector primario han generado, en las últimas décadas, una tendencia clara de emigración de población hacia las grandes urbes, provocando su paulatina despoblación (Zoido et al., 2003). La crisis económica iniciada en 2008, y su brutal impacto sobre la sociedad española, ha intensificado este éxodo generalizado de jóvenes en busca de empleo y formación en el exterior, acelerando la pérdida y envejecimiento de 
su población (Caballero et al., 2009). La recuperación económica de la "España vacia" pasa inexorablemente por una mejor gestión de los recursos naturales, destacando en el caso de Castilla y León ( $\mathrm{CyL}$ ) especialmente el potencial crecimiento y mejora en la gestión de sus montes. Según datos recogidos en el Tercer Inventario Forestal Nacional (IFN), publicado por el Ministerio de Agricultura en 2007 (Ministerio Medio Ambiente, 2007), CyL dispone de una superficie forestal arbolada de 2.982.318 Ha, lo que la posiciona como la comunidad con mayor superficie forestal de España. Destacando la explotación de las masas forestales tradicionales de coníferas para usos estructurales. Sin embargo, actualmente aún se mantienen grandes extensiones de masas forestales sin explotación directa para uso estructural, destinándose a usos secundarios de bajo rendimiento económico. Especialmente significativas las especies de reforestación como el Pinus pinaster Ait., ampliamente extendida en zonas áridas de la comunidad, y con trozas maduras susceptibles de explotación ( $>80$ años) provenientes de las políticas de reforestación de postguerra iniciada en la década de 1940 con el Plan Nacional de Repoblación (Serrada, 2000). Similar situación se plantea en especies de cultivo, como la madera de populicultura en zonas de ribera (Rueda et al., 2017). Según datos de la Junta de CyL (Junta CyL, 2014a), actualmente se están extrayendo unos 2,6 millones de $\mathrm{m}^{3} /$ año de madera, de los cuales cerca de medio millón de $\mathrm{m}^{3}\left(438.000 \mathrm{~m}^{3}\right)$ se corresponde con madera de populicultura, y $760.000 \mathrm{~m}^{3}$ de madera de pino pinaster. Su impacto económico en la sociedad es notorio, con una producción total del sector forestal maderable de CyL (producción primaria + industrial) de aproximadamente 523 millones de euros anuales (Junta CyL, 2014b), pudiéndose aumentar este impacto con una revalorización industrial.

\subsection{Uso estructural de la madera de baja calidad}

Las principales causas del desuso de estas dos especies de madera - chopo y pinaster - se deben a singularidades propias de cada especie, que limitan sus cualidades mecánicas en comparación con otros tipos de especies de madera de uso común en estructuras. El pino pinaster, especie amparada normativamente (UNE-EN 1912:2012) como madera de uso estructural en España, presenta prestaciones de gran calidad en madera seleccionada libre de singularidades, con valores de resistencia, elasticidad y densidad semejantes al resto de coníferas de uso estructural explotadas en España. Sin embargo, sus características de crecimiento y la baja o nula gestión forestal a la que se han visto sometidas tradicionalmente sus masas forestales generan trozas con fustes irregulares, de longitud limitada, gran cantidad de nudos de tamaño excesivo y la presencia recurrente de otras singularidades. Rigidez y resistencia se ven condicionadas por estas singularidades, obligando a desechar volúmenes amplios de piezas para su uso estructural, y determinando una baja rentabilidad de esta especie, al menos bajo los escasos estándares de gestión forestal a los que se han sometido las masas maduras tradicionalmente. Por su parte el chopo - especie de uso estructural históricamente en España, y de uso actual en países europeos como Francia- 
se presenta como una especie de rápido crecimiento con fustes rectos, de gran longitud, baja densidad, y propiedades mecánicas comparables a las coníferas (Casado et al., 2012). Sin embargo, debido a su anatomía, presenta unos módulos de elasticidad muy bajos, inadecuados para cumplir con las exigencias de rigidez de las normativas actuales sin recurrir a secciones de madera excesivas.

La introducción de refuerzos basados en polímeros reforzados con fibras (FRP) en la fabricación de vigas laminadas permite aumentar la resistencia y rigidez a flexión de la pieza, reduciendo la variabilidad de las propiedades mecánicas (Parvez, 2004). De esta forma, estas maderas de menor coste y prestaciones mecánicas limitadas presentan una alternativa comercial económicamente viable con secciones reforzadas de menor tamaño, peso y altas propiedades mecánicas. En la presente investigación se emplea la viga laminada tipo dúo por ser el sistema de industrialización más básico. El refuerzo FRP se introduce en la línea de encolado, en el interior de la sección durante la laminación de las vigas dúo (Fig. 1). De este modo mejora la resistencia al fuego del refuerzo en comparación con los refuerzos externos FRP, donde la exposición de la línea de encolado a las altas temperaturas limita su estabilidad (CNR-DT 201:2005). Además, esta disposición permite fabricar vigas con igual sección aparente y distintas prestaciones, variando el gramaje del refuerzo interior, sin ninguna repercusión visual. En esta investigación se analizan distintos tipos de materiales FRP, destacando la buena relación prestaciones-precio de los laminados rígidos de fibra de vidrio (GFRP) en ratios bajos de refuerzo utilizados (simple 1,07\% y doble 1,60\%).

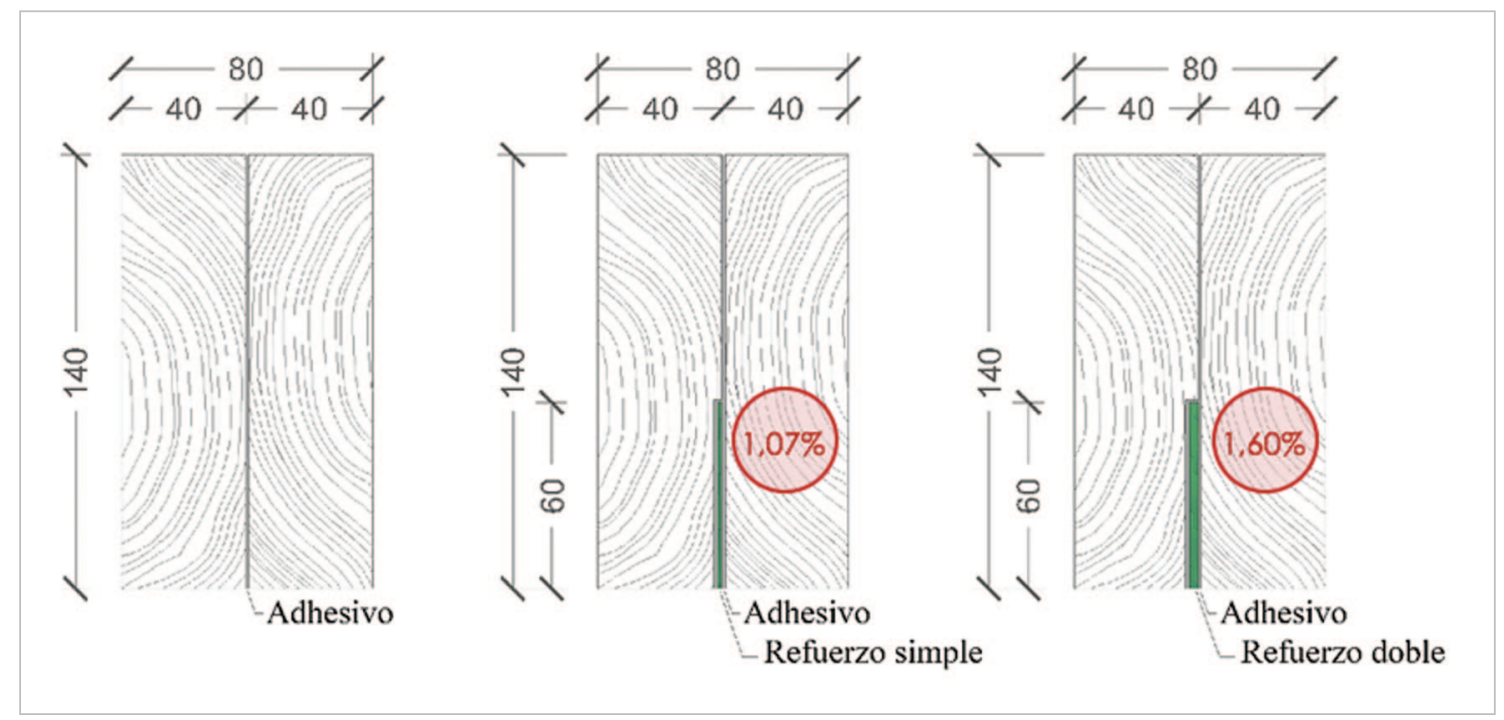

Figura 1. Secciones testigo y reforzadas de vigas dúo ensayadas.

El uso de este tipo de materiales FRP en colaboración con madera se ha aplicado de forma satisfactoria en la rehabilitación de estructuras históricas (Harte et al., 2015). Sin embargo, pese a que este tipo de soluciones se viene in- 
vestigando desde los años 60 (Bulleit, 1983) y empleando en usos de refuerzo estructural desde la década de los años 90 (Plevris, 1992), la primera investigación amplia entre distintas universidades europeas sobre el comportamiento estructural de distintos tipos de FRP, adhesivos y madera, será el proyecto GIROD (1999-2001). Más recientemente se ha desarrollado, también con fondos europeos, una completa investigación sobre su aplicación específica en el refuerzo y rehabilitación de estructuras de madera existentes a través de la COST Action FP1101 (2011-2014), tanto en la reparación de estructuras históricas como en el refuerzo de piezas nuevas de madera laminada sometidas a cargas o configuraciones con alta concentración de tensiones. Actualmente, se están llevando a cabo iniciativas a nivel europeo para la generación de nuevas guías y normativas que permitan el uso generalizado de este tipo de materiales FRP en estructuras de nueva construcción. A día de hoy, la COST Action TU1207 (2014-2017) coordina a investigadores de diversos centros de investigación con este fin, presentándose las conclusiones parciales de esta tesis en su última publicación (Basterra et al., 2016). Con todo ello, utilizar este tipo de materiales FRP para fabricar un producto compuesto que mejore, a un precio razonable, las propiedades mecánicas de las maderas de chopo y pino pinaster para su aplicación estructural, puede convertirse en una realidad.

\section{Objetivos}

La investigación que se presenta en esta Tesis Doctoral se ha desarrollado con el fin de dar un nuevo uso a especies de maderas de rápido crecimiento o con bajos niveles de calidad, bajo valor económico, y actualmente sin aplicación directa en la construcción de estructuras en España. Su introducción en el mercado como producto estructural de madera reforzada y la revalorización económica de masas forestales, pretende generar una activación de la economía local sirviendo de catalizador para la mejora de aspectos sociales y económicos de las zonas rurales.

El uso de madera estructural en edificación se ha incrementado exponencialmente, especialmente los productos de madera industrializada como madera laminada o paneles CLT, siendo la industria de la madera un sector puntero en varios países europeos. Sin embargo, de todas las especies arbóreas existentes únicamente unas pocas son utilizadas para uso estructural, y pese a emplearse en toda Europa una gestión sostenible y ordenada de las masas forestales, la creciente demanda de materia prima obligará en las próximas décadas a buscar especies alternativas con menores tiempos de rotación. El uso de maderas de rápido crecimiento, presentes de forma extensa en zonas de ribera de toda CyL, como el chopo, puede ser una alternativa viable de futuro si se logra compensar las bajas prestaciones elastomecánicas de su madera a través de procesos industriales y fabricación de secciones compuestas con otro tipo de materiales. Otra de las opciones para incrementar el volumen de materia prima para la fabricación de madera estructural pasa por optimizar los recursos forestales. Actual- 
mente, existen masas forestales cuya gestión no ha sido orientada hacia la obtención de madera para uso estructural, especialmente significativa en masas forestales de reforestación. Esta falta de tratamientos culturales adecuados con el uso final provoca unos volúmenes de madera de rechazo elevados en especies como el pino pinaster, presentes de forma extensa en zonas áridas de toda CyL. La industrialización y fabricación de secciones compuestas con otro tipo de materiales puede optimizar la utilización de los recursos madereros, permitiendo el empleo de maderas con bajas calidades para uso estructural.

Para poder dar un uso estructural real a este tipo de madera, maderas de rápido crecimiento y bajas prestaciones mecánicas o maderas de rechazo, es preciso desarrollar un producto industrializado compuesto de madera y materiales de refuerzo FRP que permita competir en prestaciones mecánicas y precio, equiparándose con otros tipos de productos de madera industrializada actualmente comercializados. No se trata tanto de fabricar un producto nuevo para las grandes líneas de laminado, como de generar un producto industrializado que pueda fabricarse fácilmente en pequeñas industrias locales, con una inversión menor, utilizando los recursos forestales cercanos, y que sea suficientemente competitivo en precio y prestaciones como para poder utilizarse en cualquier tipo de edificación. Todo ello permitiría, al menos teóricamente, realizar la segunda transformación de las materias primas en poblaciones próximas a las zonas de cultivo, generando recursos económicos ligados a los recursos forestales existentes y facilitando la creación de empleo "verde" y la fijación de población en zonas rurales.

\section{Resultados y discusión}

La investigación se divide en dos campañas experimentales; una inicial en la cual se caracterizan las propiedades mecánicas de las maderas, distintos tipos de materiales de refuerzo (FRP), y distintos tipos de adhesivos estructurales utilizados. Y una posterior, en la cual, seleccionados los refuerzos FRP y adhesivos más idóneos, se fabrican y ensayan vigas dúo reforzadas de chopo y pinaster en escala reducida (1/4) y escala real $(1 / 1)$.

Los resultados iniciales muestran como de todos los refuerzos analizados, de origen natural y sintético, la fibra de vidrio alcanza la mejor relación entre prestaciones mecánicas-precio para su empleo en vigas de madera de baja prestaciones mecánicas (Fig.2). Destacando los laminados rígidos (GFRP) por su idoneidad en la fabricación de las vigas dúo de un modo sencillo, seriado y seguro. Respecto a los adhesivos, tras la realización de ensayos a cortante y a tracción perpendicular (pull-off) sobre probetas de madera-madera y madera-GFRP, en condiciones ambientales constantes y tras ciclos de envejecimiento, destaca el buen comportamiento general de los adhesivos estructurales epoxi en todos los casos. Además, se observa como los adhesivos de poliuretano (PUR) y adhesivos de melamina-urea-formaldehído (MUF) ensayados resultan incompatibles o presentan bajas prestaciones mecánicas con los laminados GFRP utilizados. 


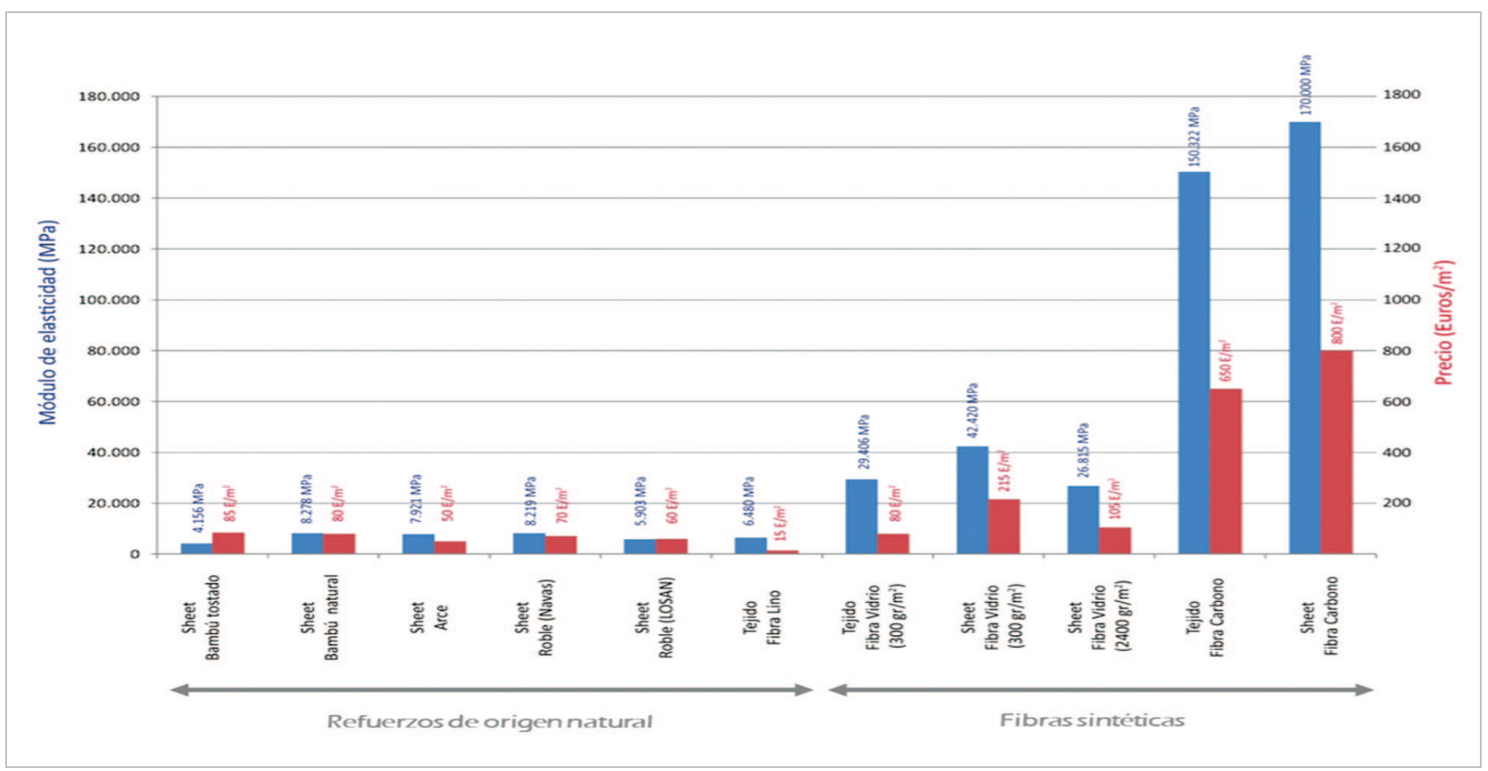

Figura 2. Comparación entre resistencia a flexión y costes de los distintos productos analizados.

Los resultados finales obtenidos sobre las vigas dúo reforzadas ensayadas a flexión (Tabla 1) muestran como, el uso de laminados rígidos de fibra de vidrio (GFRP) en ratios ligeros de refuerzo ( $<2 \%$ de la sección), colocados en la línea de encolado mejoran en todos los casos el comportamiento y homogeneidad de las vigas, reduciendo además la dispersión en las propiedades mecánicas de las vigas. Los refuerzos introducidos permiten alcanzar mejoras en rigidez (MOE) del $12-15 \%$ para las piezas de chopo, y del 7\% para pinaster. Además, permiten incrementar la resistencia última (MOR) del 25-31\% para el chopo, y del 17\% para el pinaster.

Tabla 1. Comparación de los valores de módulo de elasticidad en vigas dúo estructurales.

\begin{tabular}{l|c|c|c|c|c|c}
\hline Tipo de viga dúo & $\begin{array}{c}\text { MOE } \\
(\mathrm{MPa})\end{array}$ & $\begin{array}{c}\text { COV } \\
(\%)\end{array}$ & $\begin{array}{c}\text { Mejora } \\
(\%)\end{array}$ & $\begin{array}{c}\text { MOR } \\
(\mathrm{MPa})\end{array}$ & $\begin{array}{c}\text { COV } \\
(\%)\end{array}$ & $\begin{array}{c}\text { Mejora } \\
(\%)\end{array}$ \\
\hline Dúo chopo testigo & $\mathbf{7 . 8 3 5 , 0 2} \pm 751,89$ & 10,18 & - & $\mathbf{3 6 , 0 2} \pm 8,86$ & 25,51 & - \\
\hline $\begin{array}{l}\text { Dúo chopo reforzada } \\
\text { GFRP }\left(1200 \mathrm{gr} / \mathrm{m}^{2}\right)\end{array}$ & $\mathbf{8 . 9 1 8 , 8 7 \pm 6 9 1 , 0 9}$ & 7,60 & 12,15 & $\mathbf{4 2 , 9 5} \pm 6,92$ & 15,80 & 25,23 \\
\hline $\begin{array}{l}\text { Dúo chopo reforzada } \\
\text { GFRP }\left(2400 \mathrm{gr} / \mathrm{m}^{2}\right)\end{array}$ & $\mathbf{9 . 2 0 9 , 8 8} \pm 1.022,13$ & 10,87 & 14,73 & $\mathbf{4 6 , 0 1} \pm 7,77$ & 16,54 & 31,48 \\
\hline $\begin{array}{l}\text { Dúo pinaster testigo } \\
\text { Dúo pinaster reforzada } \\
\text { GFRP }\left(1200 \mathrm{gr} / \mathrm{m}^{2}\right)\end{array}$ & $\mathbf{1 0 . 6 8 8 , 5 3} \pm 1.599,70$ & 14,70 & - & $\mathbf{5 4 , 7 5} \pm 14,17$ & 25,41 & - \\
\hline
\end{tabular}


A partir de los resultados obtenidos anteriormente, se realiza una comparativa de la resistencia a flexión $\left(f_{m, d}\right)$ de las vigas dúos reforzadas, de chopo y pinaster, y de otras alternativas de madera, tanto aserrada como laminada, disponibles actualmente en el mercado. Partiendo en cada caso de la misma hipótesis de cargas, se calcula la sección mínima necesaria para cada producto de madera estructural y se estima su coste de acuerdo con los precios de mercado actuales, obtenidos de los catálogos comerciales de las casas comerciales suministradoras. Los resultados obtenidos de esta comparación (Fig. 3) reflejan como para las vigas dúo reforzadas con bajos ratios de GFRP $(<2 \%)$, su coste es semejante al de otras maderas laminadas, confirmándose como una solución competitiva tanto en prestaciones mecánicas como en precio.

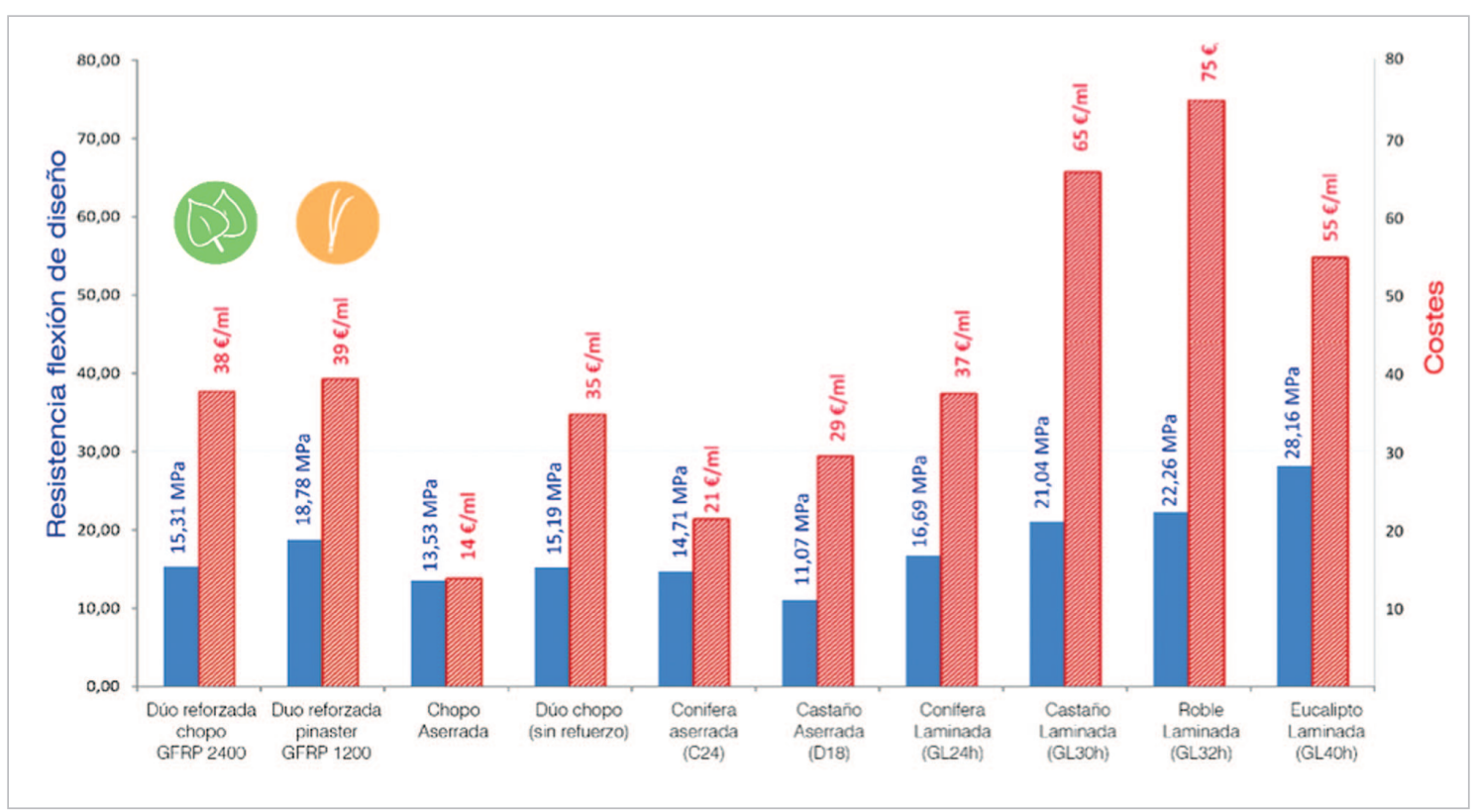

Figura 3. Comparación entre resistencia a flexión y costes de los distintos productos analizados.

Respecto al comportamiento a largo plazo o fluencia, los resultados obtenidos, tras los ensayos realizados con piezas sometidas a carga constante bajo condiciones ambientales controladas, no muestran ninguna mejora sensible y significativa ante los fenómenos de fluencia con los bajos ratios de refuerzo $(<2 \%)$ de GFRP utilizados.

\section{Conclusiones}

El uso de refuerzos GFRP en madera laminada (dúos) optimiza la utilización de los recursos madereros, al permitir emplear en la fabricación del producto estructural maderas de propiedades resistentes inferiores a las utilizadas conven- 
cionalmente, sin que ello implique una merma en las propiedades y calidad del producto final.

Las especies de madera utilizadas en esta investigación, chopo (Populus $x$ euroamericana I-214) y pino pinaster (Pinus pinaster Ait.) en calidad de rechazo, presentan unos módulos de elasticidad bajos en comparación con otras especies de madera de uso estructural habitual, lo que limita su empleo en la práctica. La fabricación de un producto industrializado, con un proceso de producción sencillo, permite, como se ha demostrado experimentalmente, aumentar las propiedades mecánicas, especialmente el módulo de elasticidad, hasta unos niveles comparables a otras maderas de uso comercial. Además, se han estudiado los costes reales de fabricación para la investigación, y estimado los costes de un producto industrializado. (Tabla 2).

Tabla 2. Resumen de las propiedades mecánicas de las vigas dúo reforzadas.

\begin{tabular}{|c|c|c|c|}
\hline & & $\begin{array}{l}\text { Viga dúo reforzada } \\
\text { de chopo } \\
\text { (GFRP UNI 2400) }\end{array}$ & $\begin{array}{l}\text { Viga dúo reforzada } \\
\text { de pino pinaster } \\
\text { (GFRP UNI 1200) }\end{array}$ \\
\hline \multicolumn{4}{|l|}{ Resistencia (característica) (N/mm²) } \\
\hline Flexión & $f_{m, g, k}$ & $22\left(28^{*}\right)$ & $24(30 * *)$ \\
\hline \multicolumn{4}{|l|}{ Rigidez (kN/mm²) } \\
\hline Módulo elasticidad paralelo medio & $\mathrm{E}_{0, \mathrm{~g}, \text { mean }}$ & 9,2 & 11,5 \\
\hline Módulo de elasticidad paralelo 5\% & $\mathrm{E}_{0, \mathrm{~g}, \mathrm{k}}$ & 7,6 & 10 \\
\hline \multicolumn{4}{|l|}{ Densidad $\left(\mathrm{kg} / \mathrm{m}^{3}\right)$} \\
\hline Densidad característica & $\rho_{\mathrm{g}, \mathrm{k}}$ & 340 & 350 \\
\hline Densidad media & $\rho_{\mathrm{g}, \text { mean }}$ & 410 & 420 \\
\hline Precio estimado & & $805 € / \mathrm{m}^{3}$ & $945 € / \mathrm{m}^{3}$ \\
\hline
\end{tabular}

De los resultados obtenidos en esta tesis doctoral se puede afirmar que es viable desarrollar vigas industrializadas de uso estructural competitivas en prestaciones y precio, a partir de maderas de rápido crecimiento y bajas prestaciones mecánicas, o a partir de maderas de rechazo de baja calidad.

\section{Financiación y agradecimientos}

Esta tesis ha estado financiada por un contrato predoctoral de la Universidad de Valladolid, dentro su programa de formación de personal docente e investigador predoctoral (Convocatoria FPI-Uva 2015). Además, para el desarrollo de la investigación presentada en esta tesis doctoral se ha contado con el apoyo económico el Ministerio de Ciencia e Innovación (BIA2012-21233) en el marco del Plan Nacional de I+D+i; y de la Junta de Castilla y León a través del programa de apoyo a proyectos de investigación cofinanciado por la Unión Europea a través del Fondo Europeo de Desarrollo Regional (VA051A10-2). 


\section{Bibliografía}

Basterra, L.A., Acuña, L., Casado, M., López, G., Morillas, L., Balmori, J.A. (2016) FRP reinforcement and production of duo laminated timber beams. Next Generation Design Guidelines for Composites in Construction [COST Action TU1207].

Brazier, J.D.; 1977. The effect of forest practices on quality of harvested crop. Forestry: An International Journal of Forest Research, 50 (1), 49-66. https://doi.org/10.1093/forestry/ 50.1 .49

Bulleit, W.M.; 1983. Reinforcement of wood materials: a review. Wood and Fiber Science, 16(3), 391-397.

Caballero, P.; Delgado, J.M.; Gómez, J.M.; González, Y.; González-Posada, E.; Niño, V.; Rueda, J.D.; Vega, C.; 2009. Perspectivas del envejecimiento activo en Castilla y León. Consejo Económico y Social de Castilla y León. Valladolid.

Casado, M.; Acuña, L.; Basterra, L.A.; Vecilla, D.; 2012. Grading of structural timber of Populus_euramericana clone I-214. Holzforschung, 66(5), 633-638. https://doi.org/10.1515/hf2011-0153

Castro, G.; Paganini, F.; 2003. Mixed glued laminated timber $\mathrm{f}$ poplar and Eucalyptus grandes clones. Holz als Roh und Werkstoff, 61 (4), 291-298. https://doi.org/10.1007/s00107-0030393-6

Harte, A.; Dietsch, P.; 2015. Reinforcement of Timber Structures. A state-of-the-art report', Shaker Verlag, Aachen.

Johansson, G.; Kliger, R.; Perstorper, M.; 1994. Quality of structural timber-product specification system required by end-users. Holz als Roh und Werkstoff, 52 (1). 42-48. https://doi. org/10.1007/BF02615017

Junta de Castilla y León, 2014(a). Crecimientos de madera y biomasa forestal en Castilla y León. Resultados. Consejería de Fomento y Medio Ambiente, Valladolid.

Junta de Castilla y León, 2014(b): Programa de movilización de los recursos forestales en Castilla y León. Consejería de Fomento y Medio Ambiente, Valladolid.

Lawson, B.; Rudder, D.; 1996. Building materials, energy and the environment: Towards ecologically sustainable development. Royal Australian Institute of Architects. Makuna, Australia.

Ministerio de Medio Ambiente, 2007. Tercer Inventario Forestal Nacional. Publicaciones del Ministerio de Medio Ambiente. Madrid.

Parvez, A.; 2004. The reinforcement of timber for structural applications and repair. $\mathrm{PhD}$ thesis, Dept Mech Eng. University of Bath.

Plevris, N.; Triantafillou, T.C.; 1992. FRP-Reinforced wood as structural material. Journal of Materials in Civil Engineering, 4(3), 300-317. https://doi.org/10.1061/(ASCE)0899-1561(19 92) 4:3(300)

Rueda, J.; García-Caballero, J.L.; Cuevas, Y.; García-Jiménez, C.; Villar C.; 2017. Cultivo de chopos en Castilla y León. Consejería de Fomento y Medio Ambiente, Junta de Castilla y León. Valladolid.

Serrada, R.; 2000. Apuntes de repoblaciones forestales. Fundación Conde del Valle de Salazar, Universidad Politécnica de Madrid.

Thelandersson, S.; Larsen, H.J.; 2003. Timber engineering. Ed. John Wiley \& Sons. Chichester, England.

Willmann, J.; Knauss, M.; Benesch, T.; Apolinarska, A.A.; Gramazio, F.; Kohler, M.; 2016. Ro- 
botic timber construction - Expanding additive fabrication to new dimensions. Automation in construction 61, 16-23. https://doi.org/10.1016/j.autcon.2015.09.011

Zoido, F.; Arroyo, A.; 2003. La población de España. Tendencias demográficas durante el siglo XX en España. Instituto Nacional de Estadística (INE). Madrid, pp. 17-75. 


\section{Datos de la tesis}

ACCESIT Premio Universitario de la SECF 2019 a la mejor tesis doctoral. Director: Luis-Alfonso Basterra Otero

Centro: Departamento de Construcciones Arquitectónicas, Ingeniería del Terreno, Mecánica de los Medios Continuos y Teoría de Estructuras. Escuela Técnica Superior de Arquitectura.

Universidad: Universidad de Valladolid

Fecha de defensa: Septiembre de 2019

\section{Publicaciones derivadas de la Tesis Doctoral}

González, Basterra, L.A.; Balmori, J.A.; Morillas, L.; Acuña, L.; Casado, M.; 2017. Internal reinforcement of laminated duo beams of low-grade timber with GFRP sheets. Construction and Building Materials 154: 914-920. https://doi.org/10.1016/ j.conbuildmat.2017.08.007

Balmori, J.A.; Basterra, L.A.; Acuña, L.; 2020. Internal GFRP reinforcement of LowGrade Maritime Pine Duo Timber Beams. Materials 13(3):571-583. https://doi. org/10.3390/ma13030571 